Man and Nature

L'homme et la nature

\title{
Le Merveilleux Parodique dans le Théâtre Forain de Lesage
}

Mary Ellen Ross

Volume 11, 1992

URI : https://id.erudit.org/iderudit/1012670ar

DOI : https://doi.org/10.7202/1012670ar

Aller au sommaire du numéro

Éditeur(s)

Canadian Society for Eighteenth-Century Studies / Société canadienne d'étude du dix-huitième siècle

ISSN

0824-3298 (imprimé)

1927-8810 (numérique)

Découvrir la revue

Citer cet article

Ross, M. E. (1992). Le Merveilleux Parodique dans le Théâtre Forain de Lesage. Man and Nature / L'homme et la nature, 11, 35-45.

https://doi.org/10.7202/1012670ar

Copyright (C Canadian Society for Eighteenth-Century Studies / Sociéte canadienne d'étude du dix-huitième siècle, 1992
Ce document est protégé par la loi sur le droit d'auteur. L'utilisation des services d'Érudit (y compris la reproduction) est assujettie à sa politique d'utilisation que vous pouvez consulter en ligne.

https://apropos.erudit.org/fr/usagers/politique-dutilisation/ 


\section{Le Merveilleux Parodique dans le Théâtre Forain de Lesage}

La démarche traditionnelle de ceux qui commentent le merveilleux forain de Lesage, c'est d'excuser l'emploi du merveilleux dans ce théâtre en faveur de la satire sociale qui caractérise les comédies foraines. C'est sans doute Lesage lui-même qui a fondé cette tradition lorsque, conscient de l'exigence classique de la vraisemblance, il tente de justifier le merveilleux forain:

On y (à l'Opéra Comique) voyait à la vérité régner ordinairement du merveilleux; mais ce merveilleux était toujours joint à des sentiments naturels et à des portraits satiriques, qui contentaient les personnes qui veulent de la morale. ${ }^{1}$

Barberet se fait l'écho de Lesage à cet égard; il note que le merveilleux permet à Lesage de supprimer des scènes de transition, pour passer plus rapidement à la satire des moeurs:

pour lui [Lesage], le merveilleux n'est pas un but, mais un moyen. C'est pour amener des scènes naturelles qu'il se place en pleine fantaisie. Il a besoin de ce large cadre pour mettre en jeu ses personnages et développer ses caractères. Il y est enfin plus à l'aise et plus libre de ses mouvements. Affranchi du soin des transitions, des ménagements, des longueurs en un mot qu'eût exigées un ordre d'événements plus près de la nature, il a pu s'épargner bien du verbiage. ${ }^{2}$

Mais les commentaires récents de Dominique Lurcel acceptent sans restriction le merveilleux de la Foire. Pour Lurcel, le merveilleux occupe en fait une place d'honneur parmi les éléments qui font l'originalité et l'intérêt des comédies foraines. Le théâtre de la Foire présente:

un mélange, unique au XVIIIe siècle, de réalisme et de merveilleux; une fantaisie que le recours au burlesque rend sans bornes; des temps et des espaces qui se télescopent; un ton constamment en ruptures, à force de gags et de clins d'yeux; 
une intrigue, tantôt quasi transparente, tantôt bousculée avec la plus franche liberté, toujours traitée au second degré: bref, les textes d'un théâtre qui, s'il n'est évidemment pas conscient de sa théatralité, tourne résolument le dos à l'illusion et qui, dans le domaine de la représentation, affirme la prééminence de tous les éléments du spectacle - danse, chant, machinerie - et du jeu de l'acteur. ${ }^{3}$

Ce sont justement ce merveilleux forain et ce refus résolu de l'illusion théâtrale qui nous intéressent dans la présente étude. Si les critiques reconnaissent que le merveilleux forain permet la satire, ils ont néanmoins très peu étudié la parodie systématique du merveilleux lui-même dans le théâtre de la Foire. ${ }^{4}$ En fait, le merveilleux forain de Lesage, tout en présentant le point de départ à des portraits satiriques, est en butte lui-même à une attaque parodique, ludique et comique.

Linda Hutcheon signale que la satire et la parodie ont souvent partie liée dans la littérature, et surtout dans la littérature du dix-huitième siècle. ${ }^{5}$ Il en va ainsi dans le théâtre forain, où le merveilleux de Lesage subit une réduction parodique, comme les moeurs de la société parisienne font l'objet d'une attaque satirique. Comme Pierre Berthiaume l'indique: 'Essentiellement parodiques, les pièces de Lesage fonctionnent selon un principe de ravalement, de réduction, qui met en pièces le pédantisme de la culture classique et les valeurs héritées de celle-ci. ${ }^{6}$ En effet la satire, phénomène social, et la parodie, phénomène plutôt formel, se côtoient dans le théâtre forain de Lesage. Comme nous le verrons, la réduction parodique du merveilleux, liée à la satire, se fait au moyen de rapprochements constants de l'exotique ou du mythologique d'une part et de la réalité française d'autre part, de jeux de langage, et de commentaires autoréflexifs qui mettent en relief le spectacle en tant que tel, brisant ainsi l'illusion du surnaturel.

Le merveilleux, où règne un surnaturel incompatible avec les lois de la réalité empirique, ${ }^{7}$ figure dans $63 \%$ des comédies foraines de Lesage. ${ }^{8}$ Ces comédies sont susceptibles d'être divisées en trois catégories générales. Il s'agit d'abord des parodies proprement dites, où le surnaturel figure dans le cadre d'une déformation comique de spectacles présentés par d'autres théâtres parisiens, et surtout par l'Opéra. Notre deuxième rubrique est celle des comédies où les éléments surnaturels prennent naissance dans un lieu exotique, que ce soit une ville orientale ou une forêt provinciale. ${ }^{9}$ On retrouve dans ces comédies exotiques des magiciens, des génies, des géants et des fées, et des objets merveilleux, tels qu'un miroir qui révèle la vertu des femmes ou une queue magique qui oblige les hypocrites à dire la vérité. Il faut compter, troisièmement, les prologues et les comédies allégoriques de Lesage, où entrent en scène des personnifications, surtout d'entités abstraites, telles que le Diable 
d'Argent. Les mêmes procédés qui, dans la parodie proprement dite, réduisent le merveilleux de façon humoristique, se retrouvent dans les comédies exotiques et dans les pièces allégoriques de Lesage, pour réduire là encore le merveilleux.

Hutcheon note que l'acte de parodier est à la fois un acte d'incorporation, où l'auteur superpose le texte parodié et le texte qui parodie, et un acte de distanciation ironique et critique. ${ }^{10}$ Dans les parodies foraines de Lesage, cette distanciation ironique par rapport au texte parodié consiste souvent, paradoxalement, en un rapprochement loufoque, qui met sur le même plan les héros ou divinités de la mythologie antique et des éléments de la réalité parisienne la plus banale. Le dieu Neptune fait ainsi la cour à Thétis dans Arlequin Thétis, parodie de l'opéra de Fontenelle: ${ }^{11}$

\section{Belle Thétis, je vous promets}

Mes maquereaux, mes harengs frais.

Il est vrai que Jupin mon frère

A pris le gros lot, sans façon;

Mais, je fais bien meilleure chère

Que lui, ma déesse, en poisson. ${ }^{12}$

La puissance du dieu des mers est ravalée au marchand de poissons qui crie ses harengs aux halles, tandis que Jupiter occupe la première place dans le panthéon romain parce qu'il a 'pris le gros lot.' Quelques scènes plus loin, le rapprochement des divinités mythologiques de l'opéra et des Parisiens du dix-huitième siècle se fait de nouveau. Le choeur chante:

Que Junon fasse la diablesse;

Thétis de Jupin est maîtresse,

Thétis a ce poste éclatant:

Mainte fille en voudrait autant.

Qu'il est doux d'être la déesse

D'un grand seigneur, ou d'un traitant! (tome 1, p. 26)

Ce passage met Jupiter au rang d'un Turcaret, riche financier qui fait des cadeaux généreux à sa maîtresse, ou, comme on le dirait de façon figurée, à sa 'déesse.'

D'autres parodies de Lesage rapprochent les êtres merveilleux de la mythologie antique et les Parisiens de l'époque, en présentant l'action même de la parodie dans un cadre parisien. Il en va ainsi dans la parodie 
d'une comédie intitulée Diane et Endymion, présentée par la Comédie Italienne. ${ }^{13}$ L'action de la parodie écrite par Lesage, Arlequin Endymion, se déroule non pas au Mont Latmos, mais à Montmartre. L'amoureuse $\mathrm{d}^{\prime}$ Endymion et la rivale de la déesse Diane est dans cette parodie une pâtissière de Montmartre, nommée Madame Tartelin.

Comme ce dernier exemple l'indique, les jeux de langage favorisent la parodie du merveilleux antique. Dans Les Amours de Protée, parodie de l'opéra de Lafont, ${ }^{14}$ le personnage mythologique Vertumne, amoureux de Pomone, déesse des fruits, chante son amour:

Ah! Pomone,
Ma mignonne,
Ma pouponne,
Quel beau jour!
Ma mignonne,
Ma pouponne,
Ma moutonne,
Quel beau jour! (tome 2, p. 182)

Le délire verbal créé par la suite de rimes, joint au caractère peu héroïque des hypocoristiques, ${ }_{15}^{15}$ servent à bafouer les deux amoureux mythologiques du théâtre rival.

Lorsqu'on passe au merveilleux exotique, on retrouve les techniques de la parodie. La comparaison rapide de La Statue merveilleuse de Lesage et de sa source, 'L'Histoire du prince Zeyn Alasnam et du roi des Génies,' tirée des Mille et Une Nuits, révèle à quel point Lesage déforme et réduit le merveilleux du conte arabe. L'action des deux ouvrages est presque identique: le prince doit trouver une fille de quinze ans, ${ }^{16}$ parfaitement belle et très chaste, pour que le roi des génies en fasse sa femme. Dans la version de Lesage, la recherche de la jeune fille vertueuse au Cachemire donne lieu, bien entendu, à une série de tiroirs satiriques, où le miroir magique du génie révèle que différentes jeunes filles ne sont pas chastes, scènes qui n'ont aucune contrepartie dans Les Mille et Une Nuits. Ainsi Pierrot compare l'une des jeunes filles en question à 'une fille d'Opéra,' remarque qui abolit l'exotisme merveilleux en faveur de la satire polémique et de la parodie. ${ }^{17}$

Mais souvent la parodie du merveilleux ne vise que le merveilleux lui-même, instaurant un jeu où le dramaturge et le spectateur acceptent de ne pas prendre au sérieux la magie. Dans un prologue autoréflexif intitulé L'Enchanteur Mirliton, deux acteurs de la Foire, Olivette et Mezzetin, se présentent à l'enchanteur Mirliton. Ils espèrent que le magicien leur donnera de nouvelles comédies à jouer. Lesage signale aux spec- 
tateurs qu'il ne faut pas trop croire à la magie du sorcier. Quand Olivette fait remarquer à l'enchanteur que ce dernier sait très bien, grâce à ses pouvoirs cabalistiques, pourquoi elle est venue le consulter, la réponse du magicien équivaut à un clin d'oeil complice qui invite le spectateur à se prêter au jeu:

Mirliton: Oui, je le sais; mais ne laissez pas de me le dire, comme si je ne le savais pas: Et je vous parlerai comme si j'ignorais ce qui se passe. Ainsi le veut le cérémonial magique. (tome 2, p. 9)

L'enchanteur met en question de façon ludique ses propres pouvoirs, tout en raillant l'une des conventions du merveilleux, à savoir la demande rituelle du suppliant qui consulte le magicien.

Les comédies exotiques de Lesage ravalent le merveilleux en le mettant systématiquement sur le plan de la réalité de tous les jours, et surtout, de la réalité parisienne de l'époque. ${ }^{18} \mathrm{Il}$ s'agit d'une démarche quelque peu paradoxale, dans la mesure où le merveilleux n'a presque jamais son origine à Paris dans les comédies foraines. ${ }^{19}$ Cependant, l'illusion de l'exotique et du merveilleux est brisée à tout moment pour permettre des allusions satiriques et parodiques. Dans La Forêt de Dodone les chênes qui étaient pendant l'antiquité les oracles de la forêt grecque se lamentent $d u$ fait que personne ne vient plus les consulter:

Fameuse forêt de Dodone,

Hélas! chacun vous abandonne!

Les hommes venaient autrefois

A genoux consulter vos chênes:

La foule à présent est aux bois

Et de Boulogne et de Vincennes. (tome 1, p. 468)

Ainsi la forêt sacrée de l'oracle subit l'empreinte dégradante des bois où les amoureux parisiens se donnent rendez-vous. De plus, on trouve parmi les personnages de cette comédie non seulement l'Arlequin et le Scaramouche de la commedia dell'arte, mais aussi des paysans nommés Colin et Colinette qui se marient, et les garçons et les filles de leur noce. Tous ces paysans ressemblent en tous points aux paysans français que Lesage peint ailleurs dans le théâtre de la Foire..$^{20}$

Lesage a recours à une stratégie des plus improbables pour relier l'action merveilleuse de Roger, roi de Sicile à la réalité française. L'action de cette comédie, située, comme l'indique le titre, en Sicile, tourne autour du sort magique qu'un magicien maléfique a jeté sur la reine de Sicile. 
Ce sort, qui empêche le roi de s'approcher de la reine, sera brisé lorsqu'on trouvera un mari qui n'est pas cocu. Or, Lisette, Parisienne travestie en Arlequin qui se fait passer pour le fou du roi à la cour de Roger, prouve sa fidélité à son mari jaloux Mezzetin. Ce dernier découvre qu'il n'est pas cocu et met ainsi fin au sort. On constate encore une fois le caractère paradoxal du merveilleux chez Lesage: si ce merveilleux prend presque toujours sa source dans un cadre autre que celui de Paris, Lesage se donne beaucoup de mal pour déconstruire le cadre exotique du merveilleux.

Comme c'était le cas dans les parodies de Lesage, les jeux de langage visent le merveilleux dans les comédies exotiques. Roger, roi de Sicile fournit un exemple assez typique de ce phénomène. Arlequin décrit à des visiteurs la façon dont le sorcier a jeté un sort sur la reine de Sicile, le jour même de ses noces avec le roi. Le magicien a essayé de séduire la reine:

Arlequin: Elle voyant cela:

Titata!

Se met fort en colère,

Et donne à l'insolent,

Patapan!

Trois soufflets pour salaire.

[...]

Le Philosophe Enchanteur,

En fureur,

Suivit sa brutale ardeur;

Et saisissant la pucelle,

Par trois fois (3 fois) souffla sur elle. (tome 2, p. 411)

L'onomatopée et le lazzi des gifles sont des éléments comiques évidents. Mais il faudrait remarquer ici le jeu de langage qui reprend le substantif 'soufflets' pour décrire comment le magicien exerce ses pouvoirs magiques, en soufflant sur la reine. C'est l'enchaînement de la réplique par la répétition des sons ('trois soufflets'/'trois fois souffla') qui gouverne en fait les détails du rituel cabalistique. Cela revient à dire que la peinture des traits du merveilleux est moins importante chez Lesage que les effets verbaux qui l'accompagnent.

Des jeux de langage parodient le merveilleux dans d'autres comédies exotiques de Lesage. L'action de la comédie Arlequin invisible ressemble de près à celle du roman Le Diable boiteux. Et, comme c'est le cas dans le roman de Lesage ${ }^{21}$ la présence du démon Asmodée permet des jeux de langage. Ainsi, Arlequin déclare qu'en comparaison d'Asmodée, les honnêtes gens 'ne valent pas le diable' (tome 1, p. 28). La parodie effectue ici une littéralisation du cliché.

La troisième catégorie regroupe les prologues et les pièces allégoriques de Lesage. L'emploi du merveilleux allégorique dans le prologue 
autoréflexif, pour exposer les intentions de l'auteur, était déjà fréquent au dix-septième siècle. ${ }^{22}$ Le merveilleux allégorique de Lesage, qui ne se limite pas aux prologues de la Foire ${ }^{23}$ sert plutôt, très souvent, à attaquer les théâtres rivaux. Il s'agit par conséquent d'un merveilleux édulcoré, qui met l'accent sur un 'autre sens' autoréflexif et polémique. ${ }^{24}$

Lesage a écrit pour la Foire une série de spectacles allégoriques qui détaillent la rivalité des divers théâtres parisiens et les procès intentés contre la Foire par la Comédie-Française. ${ }^{25}$ Les personnages allégoriques qui incarnent la Comédie-Française, la Comédie-Italienne et l'Opéra sont visés par la parodie. Dans La Querelle des théâtres la ComédieFrançaise déclame des alexandrins pour se lamenter à la Comédie-Italienne du succès de la Foire:

N'allons pas plus avant, demeurons ma mignonne.

Je ne me soutiens plus, la force m'abandonne;

Mes yeux sont étonnés du monde que je vois:

Pourquoi faut-il, hélas! qu'il ne soit pas chez moi! (tome 1, p. 272)

Ici la parodie vise le personnage 'merveilleux' allégorique et le style caractéristique du théâtre incarné par ce personnage. ${ }^{26}$

Le souci chez Lesage de bannir la distance qui sépare Paris du lieu exotique d'où provient le merveilleux est apparent dans les comédies allégoriques. L'action des pièces allégoriques se déroule en général dans un lieu vague, tel que 'la montagne sur laquelle est bâti le Temple de Mémoire. ${ }^{27}$ Cependant, les personnages qui consultent les déités allégoriques sont des types parisiens ${ }^{28}$ De même, les personnages font souvent allusion dans les pièces allégoriques à la réalité parisienne. $\mathrm{La}$ Débauche signale dans Les Routes du monde que la Grande Allée des Tuileries est l'un des lieux qu'elle préfère fréquenter (tome 2, p. 324). Et lorsque, dans La Foire des fées, des fées représentant l'argent, l'esprit, le courage et d'autres dons, organisent une Foire pour les distribuer aux êtres humains, cette Foire magique est comparée à la Foire SaintLaurent, où avaient lieu justement les spectacles forains (tome 1, p. 622).

Cette analyse nous amène naturellement à sonder la visée du procédé qui consiste dans le théâtre de la Foire à parodier le merveilleux. Il est évident que Lesage profite de ce trait pour railler ses rivaux qui se servent du merveilleux, surtout ceux de l'Opéra. Pourtant, comme on l'a vu, la cible de la parodie est souvent le merveilleux lui-même. Lesage instaure un double mouvement dans le théâtre de la Foire: le dramaturge élabore un décor exotique et une intrigue magique ou mythique, quitte à les compromettre ensuite. Les comparaisons constantes des lieux exotiques et de la France, les passages autoréflexifs, autant de clins d'oeil 
qui rappellent au spectateur qu'il assiste à un spectacle, et les jeux de langage qui rabaissent la dignité de la magie, brisent de façon parodique l'illusion du merveilleux. Ce jeu comique relève à la fois de la gaieté qui caractérise le spectacle forain et des buts satiriques de Lesage. Un merveilleux impressionnant et convaincant ne peut pas dominer les comédies de la Foire, car une telle représentation du merveilleux nuirait au comique et à la satire.

Mais le merveilleux forain représente aussi une conjoncture qui relève de deux courants parodiques. Le merveilleux chez Lesage illustre en premier lieu la tendance parodique générale du théâtre de la Foire. Pour David Trott, la poussée parodique de 'l'autre répertoire,' c'est-àdire du théâtre non-officiel de la première moitié du dix-huitième siècle, se rattache à une critique innovatrice des conventions du théâtre 'officiel. ${ }^{, 29}$ Le merveilleux de Lesage constitue effectivement une telle critique, où la parodie et l'autoréférentialité minent les institutions des théâtres rivaux.

Le merveilleux forain fait également l'objet de la parodie, rejoignant ainsi une tendance parodique du conte merveilleux du dix-huitième siècle que décrit Jacques Barchilon:

Le narrateur est un adulte qui débite des histoires 'puériles' mais qui s'en amuse sous cape, et vous le fait savoir. Car les conteurs se moquent de leurs personnages, des moeurs et des institutions de leur époque, comme ils se moquent du merveilleux avec humour. ${ }^{30}$

Jean-Paul Sermain suggère que la parodie est un trait constitutif du genre même du conte de fées littéraire des dernières années du dix-septième et du début du dix-huitième siècles. Ce trait permettrait au destinataire réel du conte de se démarquer d'un destinataire fictif qui, superstitieux et naïf, croirait au merveilleux. ${ }^{31} \mathrm{Si}$ la thèse de Sermain est juste, il serait intéressant d'approfondir la dimension parodique nécessaire du merveilleux dans le conte de fées littéraire et dans le théâtre de la Foire à cette époque.

Ainsi, le merveilleux chez Lesage ne constitue pas uniquement une source de comique. De même, le merveilleux de la Foire n'est pas un simple outil qui permet l'introduction artificielle et rapide des scènes satiriques. Le merveilleux de Lesage s'associe effectivement à la satire, mais c'est la parodie qui, démontant la façade exotique, voire l'illusion théâtrale du merveilleux lui-même, permet le triomphe forain du rire complice et de la satire.

MARY ELLEN ROSS

University of Victoria 


\section{Notes}

1 Préface des auteurs, Le Théâtre de la Foire ou l'Opéra Comique, contenant les meilleures pièces qui ont été représentées aux Foires de S. Germain et de S. Laurent, Genève, Slatkine Reprints, 1968 , tome 1, p. 8. Nous avons modernisé l'orthographe de toutes les citations.

2 V. Barberet, Lesage et le théâtre de la Foire, Genève, Slatkine Reprints, 1970, p. 74.

3 Dominique Lurcel, Introduction, Le Théâtre de la Foire au XVIIIe siècle, Paris, Union Générale d'Editions, 1983, pp. 31-32.

4 La parodie est évidemment proche parente de la satire. Cependant, la parodie vise en général une forme littéraire, tandis que la satire s'en prend à la société elle-même. (Voir Northrop Frye, Anatomy of Criticism, Princeton, Princeton University Press, 1957, pp. 233-34.)

5 Linda Hutcheon, A Theory of Parody (New York and London: Methuen, 1985), p. 51.

6 Pierre Berthiaume, 'Lesage et le spectacle forain,' Etudes françaises, vol. 15, nos 1-2, avril 1979, p. 137.

7 Voir la distinction générique entre le fantastique, le merveilleux et l'étrange proposée par Tzvetan Todorov (Introduction à la littérature fantastique, Paris, Seuil, 1970, p. 46).

8 C'est-à-dire que le merveilleux est un élément important de quarante-quatre des soixante-dix comédies foraines publiées que Marcello Spaziani attribue à Lesage seul ou à Lesage et ses collaborateurs (Marcello Spaziani, Il Teatro minore di Lesage: studi e ricerche, Roma, Angelo Signorelli, 1957). Nous n'avons pas tenu compte de ce qu'on pourrait appeler le 'faux merveilleux'; il s'agit d'épisodes dans Les Pèlerins de la Mecque (tome 2, p. 54) et dans Les Trois Commères (tome 2, pp. 517-23) que certains personnages considèrent d'abord comme relevant du merveilleux, mais qui ne violent pas en fait les lois de la réalité empirique.

9 Souvent toute l'action de la pièce se déroule en Asie. Moins souvent, Lesage situe l'action merveilleuse en province (Le Tombeau de Nostradamus, tome 1, pp. 48-56) ou dans un pays européen autre que la France (La Forêt de Dodone, tome 1, pp. 467-79). L'autre schéma préféré de Lesage prévoit qu'on confère à un personnage un don magique, dans un bois ou une forêt des alentours de Paris. Le personnage retourne ensuite à Paris pour y profiter de ses nouveaux pouvoirs merveilleux ( $L a$ Ceinture de Vénus, tome 1, pp. 78-94; Les Eaux de Merlin, tome 1, pp. 151-64).

10 Linda Hutcheon, 'Ironie et parodie: stratégie et structure,' Poétique, no 36, novembre 1978, p. 469.

11 Voir Henry Carrington Lancaster, Sunset: A History of Parisian Drama in the Last Years of Louis XIV 1701-1715, Baltimore, The Johns Hopkins Press, 1945, pp. 324-25.

12 Le Théâtre de la Foire ou L'Opéra Comique, tome 1, p. 24. Il faut noter que les couplets de la Foire, chantés sur un air populaire ou sur un air tiré de l'opéra en question, se prêtent admirablement à la parodie.

13 Le Théâtre de la Foire ou L'Opéra Comique, tome 1, p. 456.

14 Voir P.L. Jacob, éd., Bibliothèque dramatique de Monsieur de Soleinne, New York, Burt Franklin, 1967, vol. 3, p. 104. 
15 Corinne Pré rattache l'emploi des 'hypocoristiques les moins relevés' à la déchéance stylistique qui caractérise les parodies de l'Opéra Comique ('La Parodie en vaudevilles de 1715 à 1789,' Biblio 17, no. 33, 1987, 'Burlesque et formes parodiques dans la littérature et les arts,' p. 272). Pré note également le caractère autoréflexif de ces parodies, 'genre éminemment théâtral' qui joue sur 'l'illusion et la réalité de la scène' (p. 279).

16 Chez Lesage, le génie demande une fille de vingt ans.

17 Tome 1, p. 410. Lesage profite de toute occasion pour railler ses rivaux à l'Opéra, même dans les comédies foraines qui ne se présentent pas comme étant des parodies. Dans Le Tombeau de Nostradamus Arlequin travesti joue le rôle d'une femme qui désire devenir danseuse de l'Opéra et qui consulte le devin aux pouvoirs magiques. Ce prétexte permet à Lesage de faire imiter par son Arlequin la danse des actrices de l'Opéra, démarche qui réduit évidemment l'envergure du sorcier ainsi consulté (tome 1, p. 54). Dans L'Industrie, Pierrot et Jacquot descendent sur scène dans un 'char comique' qui leur permet de voler dans les airs, situation que commente Pierrot:

Pierrot: Je vois bien que tu n'aimes pas à voyager côte à côte des nuages. Cependant, mon cher petit frère Jacquot, puisque je t'ai fait recevoir à l'Opéra Comique, il faut bien que tu te fasses à la fatigue: Car, vois-tu, nous devons essuyer les mêmes corvées que les divinités de l'Opéra (tome 2, p. 305). Le procédé consiste ici à montrer les ficelles du merveilleux mécanique de l'Opéra.

18 Lesage emploie une technique similaire dans les romans qu'il situe en Espagne: la satire des moeurs 'espagnoles' semble souvent viser en réalité celles de la France. (Voir Charles Dédéyan, Lesage et 'Gil Blas,' Paris, Société d'Edition d'Enseignement Supérieur, 1965, p. 215.)

19 L'exception à cette règle serait le prologue $L^{\prime} O m b r e d u$ cocher poète, où l'enchanteur Gribouri fait apparaître l'ombre du titre sur le Pont-Neuf (tome 1, pp. 540-6).

20 Voir, à titre d'exemple, le Lucas des Amours de Nanterre (tome 1, pp. 328-43).

21 Dom Cleofas demande, lorsqu'il entend soupirer Asmodée, 'Qui diable soupire ici?' Et un peu plus tard le narrateur indique que le manteau du démon était tel 'que l'on jugeait bien qu'il fallait que le diable s'en fût mêlé' (Le Diable boiteux, éd. Roger Laufer, Paris, Mouton, 1970, pp. 86, 89).

22 Jacques Scherer, La Dramaturgie classique en France, Paris, Nizet, 1986, p. 165.

23 Quarante-trois pour cent des spectacles allégoriques de Lesage sont des pièces, plutôt que de simples prologues.

24 En fait, comme le note Todorov, toute allégorie, même celle qui n'est pas parodique et autoréflexive, constitue un danger pour la représentation convaincante du surnaturel (p. 69). Le 'merveilleux instrumental' (Todorov, p. 61) qui caractérise les premières comédies exotiques de Lesage (voir à titre d'exemple le coffre volant d'Arlequin Mahomet, 1714, tome 1, pp. 39-48) tend à se voir remplacer par le merveilleux allégorique aux années 1720 et 1730 .

25 La Querelle des théâtres (tome 1, pp. 270-6), Les Funérailles de La Foire (tome 1, pp. 355-64), Le Rappel de La Foire à la vie (tome 1, pp. 364-75), La Fausse Foire (tome 1, pp. 479-85), Les Spectacles malades (tome 2, pp. 206-13), L'Opéra Comique assiégé (tome 2, pp. 254-63). A propos des démêlés de l'Opéra Comique avec les autres théâtres parisiens à l'époque de Lesage, voir Barberet, pp. 24-9, 42-52; Maurice Albert, Les Théâtres de la Foire 1660-1789, Paris, Librairie Hachette, 1900, chapitres 3-5. 
26 Le comique verbal figure ainsi parmi les techniques parodiques du merveilleux allégorique. Mais il s'agit moins souvent de jeux de mots dans les spectacles allégoriques de Lesage, que de pastiches des styles caractéristiques des autres théâtres parisiens.

27 Le Temple de Mémoire, tome 2, p. 13.

28 Voir par exemple le procureur parisien qui consulte L'Espérance ( $L$ 'Espérance, tome 2, p. 371-2).

29 David Trott, 'French Theatre from 1700 to 1750: The "Other" Repertory,' Eighteenth-Century French Theatre: Aspects and Contexts, Magdy Gabriel Badir et David J. Langdon, éd., Edmonton, University of Alberta, 1986, pp. 32-43.

30 Jacques Barchilon, Le Conte merveilleux français de 1690 à 1790: cent ans de féerie et de poésie ignorées de l'histoire littéraire, Paris, Honoré Champion, 1975, p. xv.

31 Jean-Paul Sermain, 'La Parodie dans les contes de fées 1693-1713: une loi du genre?,' Biblio 17, no 33, pp. 541-52. 